\title{
Mycobacterium gordonae
}

National Cancer Institute

\section{Source}

National Cancer Institute. Mycobacterium gordonae. NCI Thesaurus. Code C86541.

A species of aerobic, Gram positive, rod shaped bacterium assigned to the phylum Actinobacteria. This species is acid fast, nonmotile, catalase and urease positive, negative for 3-day arylsulfatase and Tween 80 hydrolysis, and does not reduce nitrate. M. gordonae is commonly found in tap water and soil, is commensal in human mucus membranes, urine and gastric fluid and is rarely pathogenic except in immunocompromised individuals. 